\title{
Search for one large extra dimension with the DELPHI detector at LEP
}

\section{DELPHI Collaboration}

J. Abdallah ${ }^{27}$, P. Abreu ${ }^{24}$, W. Adam ${ }^{56}$, P. Adzic ${ }^{13}$, T. Albrecht ${ }^{19}$, R. Alemany-Fernandez ${ }^{10}$, T. Allmendinger ${ }^{19}$, P.P. Allport ${ }^{25}$, U. Amaldi ${ }^{31}$, N. Amapane ${ }^{49}$, S. Amato ${ }^{53}$, E. Anashkin ${ }^{38}$, A. Andreazza ${ }^{30}$, S. Andringa ${ }^{24}$, N. Anjos ${ }^{24}$,

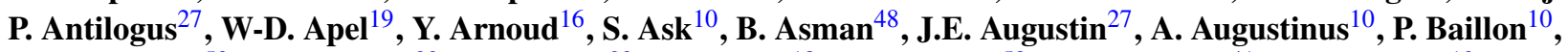
A. Ballestrero ${ }^{50}$, P. Bambade ${ }^{22}$, R. Barbier ${ }^{29}$, D. Bardin ${ }^{18}$, G.J. Barker ${ }^{58}$, A. Baroncelli ${ }^{41}$, M. Battaglia ${ }^{10}$, M. Baubillier ${ }^{27}$, K-H. Becks ${ }^{59}$, M. Begalli ${ }^{8}$, A. Behrmannn ${ }^{59}$, E. Ben-Haim ${ }^{22}$, N. Benekos ${ }^{34}$, A. Benvenuti ${ }^{6}$, C. Berat ${ }^{16}$, M. Berggren ${ }^{27}$, D. Bertrand ${ }^{3}$, M. Besancon ${ }^{42}$, N. Besson ${ }^{42}$, D. Bloch ${ }^{11}$, M. Blom $^{33}$, M. Bluj ${ }^{57}$,

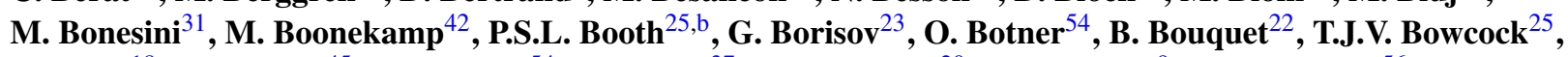
I. Boyko ${ }^{18}$, M. Bracko ${ }^{45}$, R. Brenner ${ }^{54}$, E. Brodet ${ }^{37}$, P. Bruckman ${ }^{20}$, J.M. Brunet ${ }^{9}$, B. Buschbeck ${ }^{56}$, P. Buschmann ${ }^{59}$, M. Calvi ${ }^{31}$, T. Camporesi ${ }^{10}$, V. Canale ${ }^{40}$, F. Carena ${ }^{10}$, N. Castro ${ }^{24}$, F. Cavallo ${ }^{6}$, M. Chapkin ${ }^{44}$, Ph. Charpentier ${ }^{10}$, P. Checchia ${ }^{38}$, R. Chierici ${ }^{10}$, P. Chliapnikov ${ }^{44}$, J. Chudoba ${ }^{10}$, S.U. Chung ${ }^{10}$, K. Cieslik $^{20}$, P. Collins ${ }^{10}$, R. Contri ${ }^{15}$, G. Cosme ${ }^{22}$, F. Cossutti ${ }^{51}$, M.J. Costa ${ }^{55}$, D. Crennell ${ }^{39}$, J. Cuevas ${ }^{36}$, J. D'Hondt ${ }^{3}$, T. da Silva ${ }^{53}$, W. Da Silva ${ }^{27}$,

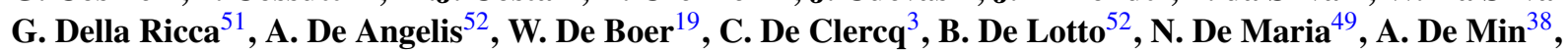

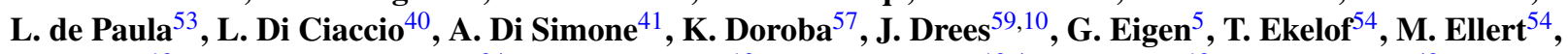
M. Elsing ${ }^{10}$, M.C. Espirito Santo ${ }^{24}$, G. Fanourakis ${ }^{13}$, D. Fassouliotis ${ }^{13,4}$, M. Feindt ${ }^{19}$, J. Fernandez ${ }^{43}$, A. Ferrer ${ }^{55}$, F. Ferro ${ }^{15}$, U. Flagmeyer ${ }^{59}$, H. Foeth ${ }^{10}$, E. Fokitis ${ }^{34}$, F. Fulda-Quenzer ${ }^{22}$, J. Fuster ${ }^{55}$, M. Gandelman ${ }^{53}$, C. Garcia ${ }^{55}$, Ph. Gavillet ${ }^{10}$, E. Gazis ${ }^{34}$, R. Gokieli ${ }^{10,57}$, B. Golob ${ }^{45,47}$, G. Gomez-Ceballos ${ }^{43}$, P. Goncalves ${ }^{24}$, E. Graziani ${ }^{41}$,

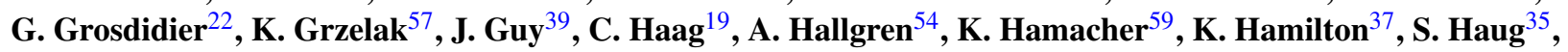
F. Hauler ${ }^{19}$, V. Hedberg ${ }^{28}$, M. Hennecke ${ }^{19}$, J. Hoffman ${ }^{57}$, S-O. Holmgren ${ }^{48}$, P.J. Holt ${ }^{10}$, M.A. Houlden ${ }^{25}$, J.N. Jackson ${ }^{25}$, G. Jarlskog ${ }^{28}$, P. Jarry ${ }^{42}$, D. Jeans ${ }^{37}$, E.K. Johansson ${ }^{48}$, P. Jonsson ${ }^{29}$, C. Joram ${ }^{10}$, L. Jungermann ${ }^{19}$, F. Kapusta ${ }^{27}$, S. Katsanevas ${ }^{29}$, E. Katsoufis ${ }^{34}$, G. Kernel $^{45}$, B.P. Kersevan $^{45,47}$, U. Kerzel $^{19}$, B.T. King ${ }^{25}$, N.J. Kjaer ${ }^{10}$, P. Kluit ${ }^{33}$, P. Kokkinias ${ }^{13}$, C. Kourkoumelis ${ }^{4}$, O. Kouznetsov ${ }^{18}$, Z. Krumstein ${ }^{18}$, M. Kucharczyk ${ }^{20}$, J. Lamsa ${ }^{1}$, G. Leder $^{56}$, F. Ledroit ${ }^{16}$, L. Leinonen ${ }^{48}$, R. Leitner ${ }^{32}$, J. Lemonne ${ }^{3}$, V. Lepeltier ${ }^{22, b}$, T. Lesiak ${ }^{20}$, W. Liebig ${ }^{59}$,

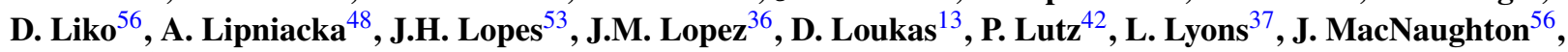

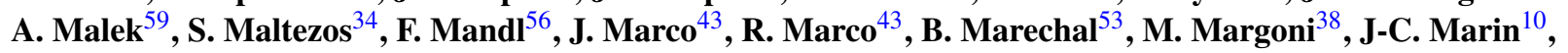
C. Mariotti $^{10}$, A. Markou ${ }^{13}$, C. Martinez-Rivero ${ }^{43}$, J. Masik $^{14}$, N. Mastroyiannopoulos ${ }^{13}$, F. Matorras ${ }^{43}$, C. Matteuzzi ${ }^{31}$, F. Mazzucato ${ }^{38}$, M. Mazzucato ${ }^{38}$, R. Mc Nulty ${ }^{25}$, C. Meroni ${ }^{30}$, E. Migliore ${ }^{49}$, W. Mitaroff ${ }^{56}$,

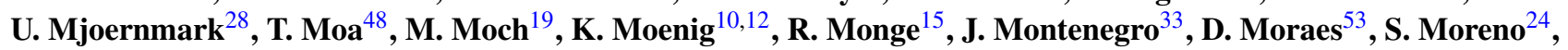
P. Morettini ${ }^{15}$, U. Mueller ${ }^{59}$, K. Muenich ${ }^{59}$, M. Mulders ${ }^{33}$, L. Mundim ${ }^{8}$, W. Murray ${ }^{39}$, B. Muryn ${ }^{21}$, G. Myatt ${ }^{37}$,

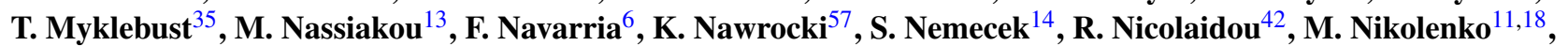
A. Oblakowska-Mucha ${ }^{21}$, V. Obraztsov ${ }^{44}$, A. Olshevski ${ }^{18}$, A. Onofre ${ }^{24}$, R. Orava ${ }^{17}$, K. Osterberg ${ }^{17}$, A. Ouraou ${ }^{42}$,

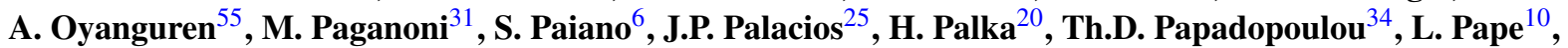

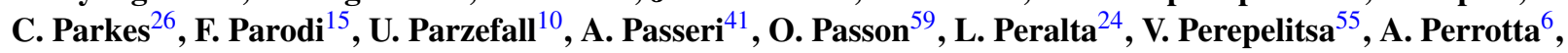

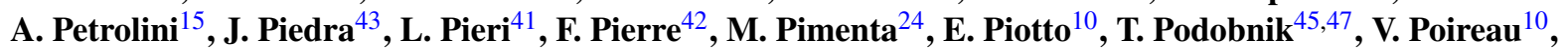
M.E. Pol ${ }^{7}$, G. Polok ${ }^{20}$, V. Pozdniakov ${ }^{18}$, N. Pukhaeva ${ }^{18}$, A. Pullia ${ }^{31}$, D. Radojicic ${ }^{37}$, P. Rebecchi ${ }^{10}$, J. Rehn ${ }^{19}$, D. Reid $^{33}$, R. Reinhardt ${ }^{59}$, P. Renton ${ }^{37}$, F. Richard ${ }^{22}$, J. Ridky ${ }^{14}$, M. Rivero ${ }^{43}$, D. Rodriguez ${ }^{43}$, A. Romero ${ }^{49}$, P. Ronchese $^{38}$, P. Roudeau ${ }^{22}$, T. Rovelli ${ }^{6}$, V. Ruhlmann-Kleider ${ }^{42}$, D. Ryabtchikov ${ }^{44}$, A. Sadovsky ${ }^{18}$, L. Salmi ${ }^{17}$, J. Salt ${ }^{55}$, C. Sander ${ }^{19}$, A. Savoy-Navarro ${ }^{27}$, U. Schwickerath ${ }^{10}$, R. Sekulin ${ }^{39}$, M. Siebel ${ }^{59}$, A. Sisakian ${ }^{18}$, G. Smadja ${ }^{29}$, O. Smirnova ${ }^{28}$, A. Sokolov ${ }^{44}$, A. Sopczak ${ }^{23}$, R. Sosnowski ${ }^{57}$, T. Spassov ${ }^{10}$, M. Stanitzki ${ }^{19}$, A. Stocchi ${ }^{22}$, J. Strauss ${ }^{56}$, B. Stugu ${ }^{5}$, M. Szczekowski ${ }^{57}$, M. Szeptycka ${ }^{57}$, T. Szumlak ${ }^{21}$, T. Tabarelli ${ }^{31}$, F. Tegenfeldt ${ }^{54}$, J. Timmermans ${ }^{33, a}$,

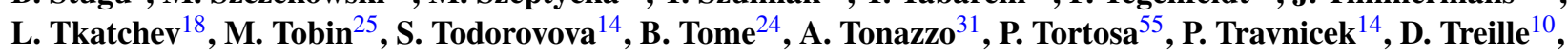
G. Tristram $^{9}$, M. Trochimczuk ${ }^{57}$, C. Troncon ${ }^{30}$, M-L. Turluer ${ }^{42}$, I.A. Tyapkin ${ }^{18}$, P. Tyapkin $^{18}$, S. Tzamarias $^{13}$, V. Uvarov ${ }^{44}$, G. Valenti ${ }^{6}$, P. Van Dam ${ }^{33}$, J. Van Eldik ${ }^{10}$, N. van Remortel ${ }^{2}$, I. Van Vulpen ${ }^{10}$, G. Vegni ${ }^{30}$, F. Veloso ${ }^{24}$, W. Venus ${ }^{39}$, P. Verdier $^{29}$, V. Verzi ${ }^{40}$, D. Vilanova ${ }^{42}$, L. Vitale ${ }^{51}$, V. Vrba ${ }^{14}$, H. Wahlen ${ }^{59}$, A.J. Washbrook ${ }^{25}$, 
C. Weiser ${ }^{19}$, D. Wicke ${ }^{10}$, J. Wickens ${ }^{3}$, G. Wilkinson ${ }^{37}$, M. Winter ${ }^{11}$, M. Witek ${ }^{20}$, O. Yushchenko ${ }^{44}$, A. Zalewska ${ }^{20}$, P. Zalewski ${ }^{57}$, D. Zavrtanik ${ }^{46}$, V. Zhuravlov ${ }^{18}$, N.I. Zimin ${ }^{18}$, A. Zintchenko ${ }^{18}$, M. Zupan ${ }^{13}$

${ }^{1}$ Department of Physics and Astronomy, Iowa State University, Ames IA 50011-3160, USA

${ }^{2}$ Physics Department, Universiteit Antwerpen, Universiteitsplein 1, 2610 Antwerpen, Belgium

${ }^{3}$ IIHE, ULB-VUB, Pleinlaan 2, 1050 Brussels, Belgium

${ }^{4}$ Physics Laboratory, University of Athens, Solonos Str. 104, 10680 Athens, Greece

${ }^{5}$ Department of Physics, University of Bergen, Allégaten 55, 5007 Bergen, Norway

${ }^{6}$ Dipartimento di Fisica, Università di Bologna and INFN, Viale C. Berti Pichat 6/2, 40127 Bologna, Italy

${ }^{7}$ Centro Brasileiro de Pesquisas Físicas, rua Xavier Sigaud 150, 22290 Rio de Janeiro, Brazil

${ }^{8}$ Inst. de Física, Univ. Estadual do Rio de Janeiro, rua São Francisco Xavier 524, Rio de Janeiro, Brazil

${ }^{9}$ Collège de France, Lab. de Physique Corpusculaire, IN2P3-CNRS, 75231 Paris Cedex 05, France

${ }^{10}$ CERN, 1211 Geneva 23, Switzerland

${ }^{11}$ Institut de Recherches Subatomiques, IN2P3-CNRS/ULP-BP20, 67037 Strasbourg Cedex, France

${ }^{12}$ DESY-Zeuthen, Platanenallee 6, 15735 Zeuthen, Germany

${ }^{13}$ Institute of Nuclear Physics, N.C.S.R. Demokritos, P.O. Box 60228, 15310 Athens, Greece

${ }^{14}$ FZU, Inst. of Phys. of the C.A.S. High Energy Physics Division, Na Slovance 2, 182 21, Praha 8, Czech Republic

${ }_{15}^{15}$ Dipartimento di Fisica, Università di Genova and INFN, Via Dodecaneso 33, 16146 Genova, Italy

${ }^{16}$ Institut des Sciences Nucléaires, IN2P3-CNRS, Université de Grenoble 1, 38026 Grenoble Cedex, France

${ }^{17}$ Helsinki Institute of Physics and Department of Physical Sciences, University of Helsinki, P.O. Box 64, 00014 Helsinki, Finland

${ }^{18}$ Joint Institute for Nuclear Research, Dubna, Head Post Office, P.O. Box 79, 101000 Moscow, Russia

${ }^{19}$ Institut für Experimentelle Kernphysik, Universität Karlsruhe, Postfach 6980, 76128 Karlsruhe, Germany

${ }^{20}$ Institute of Nuclear Physics PAN, Ul. Radzikowskiego 152, 31142 Krakow, Poland

${ }^{21}$ Faculty of Physics and Nuclear Techniques, University of Mining and Metallurgy, 30055 Krakow, Poland

${ }^{22}$ LAL, Univ Paris-Sud, CNRS/IN2P3, Orsay, France

${ }^{23}$ School of Physics and Chemistry, University of Lancaster, Lancaster LA1 4YB, UK

${ }^{24}$ LIP, IST, FCUL, Av. Elias Garcia, 14-1º, 1000 Lisboa Codex, Portugal

${ }^{25}$ Department of Physics, University of Liverpool, P.O. Box 147, Liverpool L69 3BX, UK

${ }^{26}$ Department of Physics and Astronomy, Kelvin Building, University of Glasgow, Glasgow G12 8QQ, UK

${ }^{27}$ LPNHE, IN2P3-CNRS, Univ. Paris VI et VII, Tour 33 (RdC), 4 place Jussieu, 75252 Paris Cedex 05, France

${ }^{28}$ Department of Physics, University of Lund, Sölvegatan 14, 22363 Lund, Sweden

${ }^{29}$ Université Claude Bernard de Lyon, IPNL, IN2P3-CNRS, 69622 Villeurbanne Cedex, France

${ }^{30}$ Dipartimento di Fisica, Università di Milano and INFN-MILANO, Via Celoria 16, 20133 Milan, Italy

${ }^{31}$ Dipartimento di Fisica, Università di Milano-Bicocca and INFN-MILANO, Piazza della Scienza 3, 20126 Milan, Italy

${ }^{32}$ IPNP of MFF, Charles University, Areal MFF, V Holesovickach 2, 18000 Praha 8, Czech Republic

${ }^{33}$ NIKHEF, Postbus 41882, 1009 DB Amsterdam, The Netherlands

${ }^{34}$ Physics Department, National Technical University, Zografou Campus, 15773 Athens, Greece

${ }^{35}$ Physics Department, University of Oslo, Blindern, 0316 Oslo, Norway

${ }^{36}$ Departamento de la Fisica, Universidad Oviedo, Avda. Calvo Sotelo s/n, 33007 Oviedo, Spain

${ }^{37}$ Department of Physics, University of Oxford, Keble Road, Oxford OX1 3RH, UK

${ }^{38}$ Dipartimento di Fisica, Università di Padova and INFN, Via Marzolo 8, 35131 Padua, Italy

${ }^{39}$ Rutherford Appleton Laboratory, Chilton, Didcot OX11 OQX, UK

${ }^{40}$ Dipartimento di Fisica, Università di Roma II and INFN, Tor Vergata, 00173 Rome, Italy

${ }^{41}$ Dipartimento di Fisica, Università di Roma III and INFN, Via della Vasca Navale 84, 00146 Rome, Italy

${ }^{42}$ DAPNIA/Service de Physique des Particules, CEA-Saclay, 91191 Gif-sur-Yvette Cedex, France

${ }^{43}$ Instituto de Fisica de Cantabria (CSIC-UC), Avda. los Castros s/n, 39006 Santander, Spain

${ }^{44}$ Institute for High Energy Physics, Serpukov P.O. Box 35, Protvino, Moscow Region, Russia

45 J. Stefan Institute, Jamova 39, 1000 Ljubljana, Slovenia

${ }^{46}$ Laboratory for Astroparticle Physics, University of Nova Gorica, Kostanjeviska 16a, 5000 Nova Gorica, Slovenia

${ }^{47}$ Department of Physics, University of Ljubljana, 1000 Ljubljana, Slovenia

${ }^{48}$ Fysikum, Stockholm University, Box 6730, 11385 Stockholm, Sweden

${ }^{49}$ Dipartimento di Fisica Sperimentale, Università di Torino and INFN, Via P. Giuria 1, 10125 Turin, Italy

${ }^{50}$ INFN, Sezione di Torino and Dipartimento di Fisica Teorica, Università di Torino, Via Giuria 1, 10125 Turin, Italy

${ }_{52}^{51}$ Dipartimento di Fisica, Università di Trieste and INFN, Via A. Valerio 2, 34127 Trieste, Italy

${ }^{52}$ Istituto di Fisica, Università di Udine and INFN, 33100 Udine, Italy

${ }^{53}$ Univ. Federal do Rio de Janeiro, C.P. 68528, Cidade Univ., Ilha do Fundão, 21945-970 Rio de Janeiro, Brazil

${ }_{55}^{54}$ Department of Radiation Sciences, University of Uppsala, P.O. Box 535, 75121 Uppsala, Sweden

${ }_{55}^{55}$ IFIC, Valencia-CSIC, and DFAMN, U. de Valencia, Avda. Dr. Moliner 50, 46100 Burjassot (Valencia), Spain

${ }^{56}$ Institut für Hochenergiephysik, Österr. Akad. d. Wissensch., Nikolsdorfergasse 18, 1050 Vienna, Austria

${ }^{57}$ Institute Nuclear Studies and University of Warsaw, Ul. Hoza 69, 00681 Warsaw, Poland

${ }^{58}$ University of Warwick, Coventry CV4 7AL, UK

${ }^{59}$ Fachbereich Physik, University of Wuppertal, Postfach 100 127, 42097 Wuppertal, Germany 
Abstract Single photons detected by the DELPHI experiment at LEP2 in the years 1997-2000 are reanalysed to investigate the existence of a single extra dimension in a modified ADD scenario with slightly warped large extra dimensions. The data collected at centre-of-mass energies between 180 and $209 \mathrm{GeV}$ for an integrated luminosity of $\sim 650 \mathrm{pb}^{-1}$ agree with the predictions of the Standard Model and allow a limit to be set on graviton emission in one large extra dimension. The limit obtained on the fundamental mass scale $M_{D}$ is $1.69 \mathrm{TeV} / c^{2}$ at $95 \% \mathrm{CL}$, with an expected limit of $1.71 \mathrm{TeV} / c^{2}$.

\section{Introduction}

The Standard Model (SM) has been thoroughly tested at the CERN LEP $e^{+} e^{-}$collider $[1,2]$. No sign of statistically significant deviations from it or evidence for new physics phenomena beyond it have been found up to the highest LEP centre-of-mass energies of about $209 \mathrm{GeV}$. Yet the SM cannot be the final picture, because of several theoretical problems. One is known as the hierarchy problem and is related to the observed weakness of gravity in comparison with other interactions. This may be expressed by the observation that the reduced Planck mass, $M_{P l}=\sqrt{1 / G_{N}} \sim$ $2.4 \times 10^{15} \mathrm{TeV} / c^{2}$, where $G_{N}$ is Newton's coupling constant, is much larger than the $0.1-1 \mathrm{TeV} / c^{2}$ scale of the electroweak symmetry breaking.

A step towards the solution of this puzzle was proposed in 1998 by Arkani-Hamed, Dimopoulos and Dvali (ADD) [3], assuming the existence of large extra spatial dimensions (ED). Models with one ED were proposed a long time ago in connection with gravity and its unification with electromagnetism in the papers of Kaluza and Klein (KK) [4-6]. More recently, with the appearance of string theory, the existence of several EDs was advocated, but their size was thought to be close to the Planck length, $R \sim 1 / M_{P l} \sim 10^{-33} \mathrm{~cm}$. In this case EDs would be completely out of the reach of present and planned colliders. The novel suggestion of ADD was the possible existence of large EDs with a fundamental Planck mass close to the electroweak scale, in fact implying that non-trivial physics "ends" at energies of about $1 \mathrm{TeV}$. In the ADD model all the SM particles are supposed to live on a 3D brane corresponding to our usual space, while gravitons are allowed to propagate into the bulk. Thus the weakness of gravity is simply due to its dilution in the volume of the EDs.

Assuming flat EDs and compactification on a torus, Gauss' law gives:

$$
M_{P l}^{2}=R^{n} M_{D}^{n+2}
$$

\footnotetext{
a e-mail: jan.timmermans@cern.ch

${ }^{b}$ Deceased.
}

where $R$ is the radius of the ED and $M_{D}$ is the fundamental Planck scale in the D-dimensional space-time $(D=4+n)$. With $M_{D} \sim 1 \mathrm{TeV} / c^{2}$ and $n=1$, (1.1) implies a modification of Newton's law over solar system distances which is not observed. So the possibility that $n=1$ is usually considered to be falsified. On the other hand for $n \geq 2, R<1 \mathrm{~mm}$ and tests of gravity are only recently reaching these small distances [7]. For $n \geq 3, R<1 \mathrm{~nm}$ and no gravity test exists which can falsify the model.

The graviton, confined within flat EDs of size $R$, has a uniform spectrum of excitations, which, from the point of view of a $4 \mathrm{D}$ observer, will be seen as a KK tower of states, with masses uniformly spaced between $1 / R$ $\left(\sim 10^{-32 / n} \mathrm{TeV} / c^{2}\right)$ and $M_{D}$. In particle collisions at accelerators and in the cosmos, gravitons can be emitted, but they escape immediately into the bulk, with momentum conservation in all the dimensions, and are therefore detectable via a missing energy signature. Each KK state is very weakly coupled, yet the number of states is very large, which turns into a sizable cross-section for graviton emission. Astrophysics yields strong constraints for $n=2,3$ based on observations of supernova SN1987A and on the behavior of neutron stars $[8,9]$. The limits on the $M_{D}$ scale vary from 20 to $40 \mathrm{TeV} / c^{2}$ and 2 to $3 \mathrm{TeV} / c^{2}$, respectively, and seem to rule out the ADD model with $M_{D}=1 \mathrm{TeV} / c^{2}$. They are however based on many assumptions with differences of a factor of 2-3 between different calculations. For larger $n$ they become much weaker.

For $n \geq 2$ limits on graviton emission have been obtained at the LEP collider [10-13] and at the Tevatron [14, 15]. At LEP the direct graviton emission reaction $e^{+} e^{-} \rightarrow G \gamma$ $(G Z)$ has been studied: for $n \geq 2$ the photon spectrum peaks at low energies and at small emission angles [16]. No excess with respect to the SM predictions has been found and a combination of the LEP results yielded $M_{D}>1.60$ (0.80) $\mathrm{TeV} / c^{2}$ for $n=2(6)$ at the $95 \%$ Confidence Level (CL) [17].

Recently the ADD model has been reconsidered by Giudice, Plehn and Strumia (GPS) [18], who have focused on the infrared (IR) behavior of the model in connection with limits at colliders versus gravity and astrophysics constraints. They considered a distorted version of the ADD model with the same properties in the ultraviolet (UV) region, but satisfying observational and astrophysical limits in the large distance regime. They showed that the introduction of an IR cut-off in the ADD model evades the constraints from astrophysics and gravity for small $n$, including $n=1$, given the energy resolution of the collider experiments. This IR cut-off is equivalent to a slight deformation or warping of the otherwise flat EDs. They started from the Randall and Sundrum type 1 model (RS1) [19] and considered the limit of slightly warped but large ED, resulting in a moderately large total warp factor. In RS1 the visible brane is located 
at $y=0$, where $y$ is the coordinate in the extra dimension, and the Planck brane at $y=\pi R$. The line element is nonfactorisable due to the warping factor

$d s^{2}=e^{2 \sigma(y)} \eta_{\mu \nu} d x^{\mu} d x^{\nu}+d y^{2}$

with $\sigma(y)=\mu|y|$. Here $\mu$ is a mass parameter due to the warp, which has a value $50 \mathrm{MeV} / c^{2} \leq \mu \ll 1 \mathrm{TeV} / c^{2}$ and introduces an IR cut-off. This cut-off implies a mass of the graviton which is inaccessible for cosmological processes, but which has no significant implications for the high energy collider signal in the UV region of the KK spectra. In particular, the relation between the fundamental mass scale in 5 dimensions and the 4D Planck mass becomes

$M_{P l}^{2}=\frac{M_{5}^{3}}{2 \mu \pi}\left(e^{2 \mu R \pi}-1\right)$,

where $R$ is the radius of the compactified ED. Hence the one ED can still be large, but unobserved as a modification of Newton's law or in the cosmological low energy processes. In this model the hierarchy between the Fermi and Planck scales is generated by two factors, the large ED and warping. It can be seen that for $\mu \ll R^{-1}$ the ADD limit, (1.1), is obtained.

Since a search for graviton emission with $n=1$ was not performed in the previous publication [11] and since the results cannot be inferred from the limits already given for $n \geq 2$ because the photon energy spectra differ noticeably for different values of $n[16,18]$, the DELPHI data were reanalysed and the results will be presented here. The paper is organized as follows: Sect. 2 recalls briefly the experimental details, the analysis is discussed in Sect. 3, Sect. 4 presents the results and the conclusions are given in Sect. 5.

\section{Detector and data preselection}

The general criteria for the selection of single-photon events are based mainly on the electromagnetic calorimeters and on the tracking system of the DELPHI detector [20, 21]. All the three major electromagnetic calorimeters in DELPHI, the High density Projection Chamber (HPC), the Forward ElectroMagnetic Calorimeter (FEMC) and the Small angle TIle Calorimeter (STIC), have been used in the singlephoton reconstruction. The STIC accepted photons at very small polar angle, ${ }^{1}$ the FEMC covered intermediate angles, and large angles with respect to the beams were covered by the HPC. Hermeticity Taggers were used to ensure detector hermeticity for additional neutral particles in the angular region around $45^{\circ}$ between HPC and FEMC, not covered by

\footnotetext{
${ }^{1}$ In the DELPHI coordinate system, the $z$ axis is along the electron beam direction and the polar angle to the $z$ axis is called $\theta$.
}

the calorimeters. The DELPHI tracking system and the taggers were used as a veto. A detailed description of the trigger conditions and efficiencies of the calorimeters is given in a previous publication [11], where the rejection of events in which charged particles were produced is also discussed.

The study was done with data taken during the 19972000 runs at $e^{+} e^{-}$centre-of-mass energies from 180 to $209 \mathrm{GeV}$, corresponding to an integrated luminosity of $\sim 650 \mathrm{pb}^{-1}$, with the subdetectors relevant for the analysis all fully operational.

The single-photon events were selected in two stages. In the first stage events with only one detected photon were preselected and compared to the SM process $e^{+} e^{-} \rightarrow \nu \bar{v} \gamma$. A likelihood ratio method was then used to maximize the sensitivity in the search for graviton production with $n=1$.

Events with a photon in the HPC were selected by requiring a shower having a scaled energy $x_{\gamma}=E_{\gamma} / E_{\text {beam }}>$ $0.06, \theta$ between $45^{\circ}$ and $135^{\circ}$, and no charged particle tracks. Photons in the FEMC were required to have a scaled energy $x_{\gamma}>0.10$ and a polar angle in the intervals $12^{\circ}<$ $\theta<32^{\circ}\left(148^{\circ}<\theta<168^{\circ}\right)$. Single photons in the STIC were preselected by requiring one shower with a scaled energy $x_{\gamma}>0.30$ and with $3.8^{\circ}<\theta<8^{\circ}\left(172^{\circ}<\theta<\right.$ $\left.176.2^{\circ}\right)$. Additional details about the preselection are given in [11]. In the single-photon event preselection events with more than one photon were accepted only if the other photons were at low angle $\left(\theta_{\gamma}<2.2^{\circ}\right)$, low energy $\left(E_{\gamma}<\right.$ $0.8 \mathrm{GeV}$ ) or within $3^{\circ}, 15^{\circ}, 20^{\circ}$ from the highest energy photon in the STIC, FEMC and HPC respectively.

\section{Single-photon analysis}

The single-photon analysis has been discussed in detail in [11], here we will recall the main points and underline the differences in the present analysis.

Single-photon events can be faked by the QED reaction $e^{+} e^{-} \rightarrow e^{+} e^{-} \gamma$ if the two electrons escape undetected along the beampipe or if the electrons are in the detector acceptance but are not detected by the experiment. This process has a very high cross-section, decreasing rapidly with increasing energy and polar angle of the photon. Its behavior together with the rapid variation of efficiencies at low photon energy motivates the different calorimeter energy cuts in the preselection and additional energy-dependent cuts on the polar angle in the FEMC and STIC.

The remaining background from the $e^{+} e^{-} \rightarrow e^{+} e^{-} \gamma$ process was calculated with the Monte Carlo program TEEG by D. Karlen [22] and two different event topologies were found to contribute, giving background at low and high photon energy respectively. Either both electrons were below the STIC acceptance or one of the electrons was in the DELPHI acceptance where it was wrongly identified as a pho- 
ton, while the photon was lost for example in the gaps between the electromagnetic calorimeters not covered by the Hermeticity Taggers, or in masked crystals in the FEMC.

The contribution from other processes has also been calculated: cosmic ray events, $\gamma \gamma$ collisions using PYTHIA 6.1 [23] and $\mathrm{BDK}[24,25], e^{+} e^{-} \rightarrow \gamma \gamma(\gamma)$ according to Berends et al. [26-28], $e^{+} e^{-} \rightarrow \mu \mu(\gamma)$ and $e^{+} e^{-} \rightarrow \tau \tau(\gamma)$ with KORALZ [29, 30], and four-fermion events with EXCALIBUR [31] and Grc4f [32].

The $e^{+} e^{-} \rightarrow \nu \bar{v} \gamma(\gamma)$ process was simulated by the KORALZ [29, 30] program. A comparison of the crosssection predicted by KORALZ 4.02 with that predicted by NUNUGPV [33, 34] and KK 4.19 [35] showed agreement at the percent level. This difference is negligible with respect to the statistical and systematic uncertainties in the present measurement.

Simulated events for the irreducible contribution from $\nu \bar{v} \gamma$ production and other SM backgrounds were generated at the different centre-of-mass energies and passed through the full DELPHI simulation and reconstruction chain [20, 21].

Figure 3.1 shows the $x_{\gamma}$ distribution of all preselected single-photon events. As discussed in the previous paper [11], only single photon events in the HPC and FEMC were used for the subsequent analysis, since the $E_{\gamma}$ cuts in the STIC, needed to reduce the radiative Bhabha background, reject a large part of the ED signal even in the case $n=1$.

Table 3.1 shows the total number of observed and expected events in the HPC and FEMC. The numbers are integrated over the LEP energies from 180 to $209 \mathrm{GeV}$ and correspond to an overall luminosity of $\sim 650 \mathrm{pb}^{-1}$.

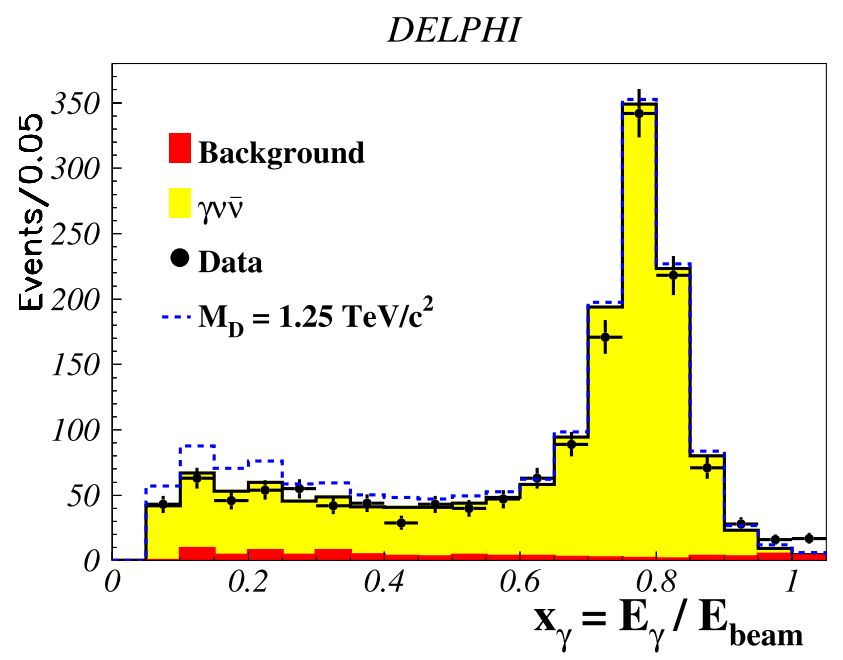

Fig. 3.1 $x_{\gamma}$ of selected single photons. The light shaded area is the expected distribution from $e^{+} e^{-} \rightarrow \nu \bar{\nu} \gamma(\gamma)$ and the dark shaded area is the total background from other sources. Indicated in the plot is also the signal expected from $e^{+} e^{-} \rightarrow G \gamma$ for $n=1$ and $M_{D}=1.25 \mathrm{TeV} / c^{2}$
A likelihood ratio method was used to select the final sample of single-photon events. This method allows the final selection to be optimized for excluding the cross section of a given signal assuming that no signal is present in the data sample. Hence the method optimizes the background suppression for a given signal efficiency [36]. The likelihood ratio function used in this analysis is given by:

$\mathcal{L}_{R}=\frac{\mathcal{L}_{S}}{\mathcal{L}_{B}}=\frac{P_{S}\left(E_{\gamma}\right)}{P_{B}\left(E_{\gamma}\right)}$.

The probability density functions $\left(P_{i=S, B}\right)$ used to construct $\mathcal{L}_{R}$ were produced from the normalized photon energy distributions of the expected ED and SM background events, after passing through the same selection criteria. A low pass filter was also used to eliminate the high frequency statistical fluctuations from the final $P_{i}$ functions. An event was then selected as a candidate event if it passed the requirement $\mathcal{L}_{R}>\mathcal{L}_{R}^{C U T}$. The value of $\mathcal{L}_{R}^{C U T}$ was determined by minimizing the expected excluded cross section in the absence of a signal:

$\sigma^{\min }\left(\mathcal{L}_{R}^{C U T}\right)=\frac{N_{95}^{\min }\left(\mathcal{L}_{R}^{C U T}\right)}{\epsilon^{\max }\left(\mathcal{L}_{R}^{C U T}\right) \times L}$,

where $N_{95}^{\min }$ is the upper limit on the number of signal events at $95 \%$ CL computed with the mono-channel version of the Bayesian method in [37]. $\epsilon^{\max }$ is the efficiency for the signal and $L$ is the integrated luminosity. The values of $N_{95}^{\min }$ and $\epsilon^{\max }$ both decrease with an increasing value of $\mathcal{L}_{R}^{C U T}$. Their derivatives, however, behave differently which results in a well defined minimum of $\sigma^{\min }\left(\mathcal{L}_{R}^{C U T}\right)$.

The data collected at different centre-of-mass energies were analysed separately and different analyses were made depending on the electromagnetic calorimeter in which the photon was recorded. The $\mathcal{L}_{R}^{C U T}$ values obtained showed a variation of around $0.7 \pm 0.1$, though all the final selections contained a rejected region in the energy spectra that covered most of the $Z$-peak, as expected. In some cases the selection also implied slightly stronger criteria for the overall minimum photon energy. Out of the preselected FEMC events, 262 passed the final selection with 250.6 expected and from the HPC events, 255 were selected with 263.5 expected. The signal efficiency of the final selection was between $85 \%$ and $90 \%$ with respect to preselection level. The final experimental limit was obtained using a Bayesian multi-channel method [37] which combined the results of

Table 3.1 The number of selected and expected single-photon events

\begin{tabular}{llll}
\hline & $N_{\text {observed }}$ & $N_{e^{+} e^{-} \rightarrow \nu \bar{v}(\gamma)}$ & $N_{\text {other SM background }}$ \\
\hline FEMC & 705 & $626 \pm 3$ & 49.1 \\
HPC & 498 & $540 \pm 4$ & 0.6 \\
\hline
\end{tabular}


the 20 analyses, the data for the two calorimeters being grouped into 10 datasets between 180 and $209 \mathrm{GeV}$ centreof-mass energy. The method takes into account all the available information (such as the fraction of the signal and the average background in each subdetector and in each data subsample) in order to properly calculate the final limit.

\section{Limit on the production of gravitons}

The differential cross-section for $e^{+} e^{-} \rightarrow G \gamma$ has been calculated in $[16,18]$ and is given by:

$$
\frac{d^{2} \sigma}{d x_{\gamma} d \cos \theta_{\gamma}}=\frac{\alpha}{32 s} \frac{\pi^{\frac{n}{2}}}{\Gamma\left(\frac{n}{2}\right)}\left(\frac{\sqrt{s}}{M_{D}}\right)^{n+2} f\left(x_{\gamma}, \cos \theta_{\gamma}\right),
$$

with

$$
\begin{aligned}
f(x, y)= & \frac{2(1-x)^{\frac{n}{2}-1}}{x\left(1-y^{2}\right)}\left[(2-x)^{2}\left(1-x+x^{2}\right)\right. \\
& \left.-3 y^{2} x^{2}(1-x)-y^{4} x^{4}\right] .
\end{aligned}
$$

Initial state radiation can produce additional photons that would cause a signal event to be rejected in a single-photon analysis. The expected signal cross-section has therefore been corrected with a radiator approximation method [38].

For $n>1$ the differential distribution, (4.2), is peaked at small $E_{\gamma}$ and $\theta_{\gamma}$, for $n=1$ instead a singularity is present at $x_{\gamma}=1$, which makes the distribution qualitatively different from the others. For instance the ratio of the cross-sections, (4.1) and (4.2), for $n=1$ and $n=2$ is independent of $\theta_{\gamma}$, and increases from $\sim 1.5$ at small $x_{\gamma}$ to $\sim 22$ at $x_{\gamma}=0.995$ for $M_{D}=1 \mathrm{TeV} / c^{2}$ and $\sqrt{s}=208 \mathrm{GeV}$. In order to take into account detector effects, the theoretical ED cross-section has been corrected for efficiency and energy resolution in the calorimeters, using a parameterization developed in the $v \bar{v} \gamma$ analysis. The theoretical energy distributions for $n=1$ and 2 smeared in the HPC and FEMC are shown in Fig. 4.1.

As can be seen in Fig. 3.1, the single photon data measured by DELPHI were well compatible with expectations from SM processes and no evidence for graviton production was found.

All DELPHI data with $\sqrt{s}>180 \mathrm{GeV}$ were used and a dedicated selection for each bin in $\sqrt{s}$ was made as described in the previous section. These results were combined to give a $95 \%$ CL cross-section limit for one extra dimension of $0.171 \mathrm{pb}$ at $208 \mathrm{GeV}$, with an expected limit of $0.166 \mathrm{pb}$. In terms of the parameter $p=\left(1 / M_{D}\right)^{3}$, to which the $n=1$ signal cross-section is proportional, the combined loglikelihood function of the Bayesian formula was practically parabolic. $p$ is estimated to be $(0.009 \pm 0.098)\left(\mathrm{TeV} / c^{2}\right)^{-3}$ and is therefore consistent with zero. The obtained limit on the fundamental mass scale is $M_{D}>1.69 \mathrm{TeV} / c^{2}$ at $95 \%$

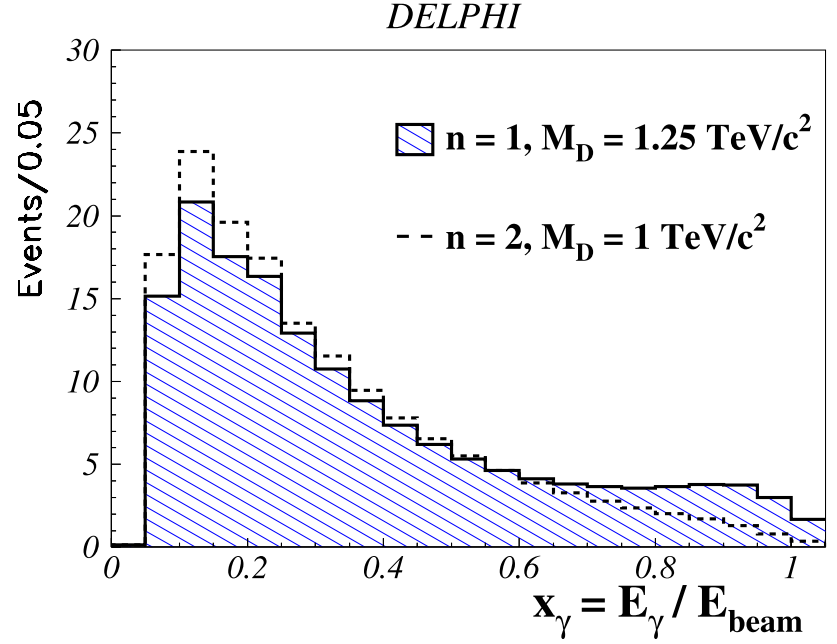

Fig. 4.1 $x_{\gamma}$ of expected single photons in the HPC and FEMC from $e^{+} e^{-} \rightarrow G \gamma$ with $n=1, M_{D}=1.25 \mathrm{TeV} / c^{2}$ and $n=2$, $M_{D}=1 \mathrm{TeV} / c^{2}$, corrected for calorimeter efficiency and resolution. $\mathrm{MC}$ expectations are normalized to the luminosity of the combined data set in Fig. 3.1

CL (with $1.71 \mathrm{TeV} / c^{2}$ expected limit) in the $n=1$ analysis. As a comparison, the cross-section limits in the previous analysis for $n=2-6$ varied between 0.14 and $0.18 \mathrm{pb}$, and the obtained limits for $M_{D}$ between $1.31 \mathrm{TeV} / c^{2}(n=2)$ and $0.58 \mathrm{TeV} / c^{2}(n=6)$. Since the characteristic peak of the $n=1$ photon spectrum at $x_{\gamma}=1$ is less prominent after including detector effects, the cross section limit is similar to those obtained for $n>1$. The same systematic errors were considered as in the previous analysis [11], namely trigger and identification efficiency, calorimeter energy scale and background, and the effect on the $M_{D}$ limit from the systematic errors in the $n=1$ analysis was estimated to be less than $4 \%$.

\section{Conclusions}

We have re-analysed single-photon events detected with DELPHI at LEP2 during 1997-2000 at centre-of-mass energies between 180 and $209 \mathrm{GeV}$ to study graviton production with $n=1$ large extra dimensions, motivated by the model of Giudice, Plehn and Strumia [18]. Since the measured single-photon cross-sections are in agreement with the expectations from the SM process $e^{+} e^{-} \rightarrow \nu \bar{v} \gamma(\gamma)$, the absence of an excess of events has been used to set a limit of $1.69 \mathrm{TeV} / c^{2}$ at $95 \% \mathrm{CL}$ on the fundamental mass scale for $n=1 \mathrm{ED}$.

Acknowledgements We are greatly indebted to our technical collaborators, to the members of the CERN-SL Division for the excellent performance of the LEP collider, and to the funding agencies for their support in building and operating the DELPHI detector. We acknowledge in particular the support of Austrian Fed- 
eral Ministry of Education, Science and Culture, GZ 616.364/2III/2a/98, FNRS-FWO, Flanders Institute to encourage scientific and technological research in the industry (IWT) and Belgian Federal Office for Scientific, Technical and Cultural affairs (OSTC), Belgium, FINEP, CNPq, CAPES, FUJB and FAPERJ, Brazil, Ministry of Education of the Czech Republic, project LC527, Academy of Sciences of the Czech Republic, project AV0Z10100502, Commission of the European Communities (DG XII), Direction des Sciences de la Matière, CEA, France, Bundesministerium für Bildung, Wissenschaft, Forschung und Technologie, Germany, General Secretariat for Research and Technology, Greece, National Science Foundation (NWO) and Foundation for Research on Matter (FOM), The Netherlands, Norwegian Research Council, State Committee for Scientific Research, Poland, SPUB-M/CERN/PO3/DZ296/2000, SPUBM/CERN/PO3/DZ297/2000, 2P03B 10419 and 2P03B 69 23(20022004), FCT—Fundação para a Ciência e Tecnologia, Portugal, Vedecka grantova agentura MS SR, Slovakia, Nr. 95/5195/134, Ministry of Science and Technology of the Republic of Slovenia, CICYT, Spain, AEN99-0950 and AEN99-0761, The Swedish Research Council, The Science and Technology Facilities Council, UK, Department of Energy, USA, DE-FG02-01ER41155, EEC RTN contract HPRN-CT00292-2002.

\section{References}

1. The ALEPH, DELPHI, L3, OPAL, SLD Collaborations, the LEP Electroweak Working Group, the SLD Electroweak and Heavy Flavour Groups, Phys. Rep. 427, 257 (2006)

2. The LEP Collaborations ALEPH, DELPHI, L3, OPAL, the LEP Electroweak Working Group, Precision electroweak measurements and constraints on the Standard Model, CERN-PHEP/2007-039 (2007). arXiv:0712.0929 [hep-ex]

3. N. Arkani-Hamed, S. Dimopoulos, G. Dvali, Phys. Lett. B 429, 263 (1998)

4. Th. Kaluza, Sitzungber. Preuss. Akad. Wiss. Phys. Math. Klasse $966(1921)$

5. O. Klein, Z. Phys. 37, 895 (1926)

6. O. Klein, Nature 118, 516 (1926)

7. C.D. Hoyle et al., Phys. Rev. D 70, 042004 (2004)

8. C. Hanhart et al., Phys. Lett. B 509, 1 (2001)

9. M. Casse et al., Phys. Rev. Lett. 92, 111102 (2004)

10. A. Heister et al. (ALEPH Collaboration), Eur. Phys. J. C 28, 1 (2003)
11. J. Abdallah et al. (DELPHI Collaboration), Eur. Phys. J. C 38, 395 (2005)

12. P. Achard et al. (L3 Collaboration), Phys. Lett. B 587, 16 (2004)

13. G. Abbiendi et al. (OPAL Collaboration), Eur. Phys. J. C 18, 253 (2000)

14. D. Acosta et al. (CDF Collaboration), Phys. Rev. Lett. 92, 121802 (2004)

15. V.M. Abazov et al. (D0 Collaboration), Phys. Rev. Lett. 90, $251802(2003)$

16. G.F. Giudice, R. Rattazzi, J.D. Wells, Nucl. Phys. B 544, 3 (1999)

17. ALEPH, DELPHI, L3, OPAL Collaborations and the LEP Exotica Working Group, LEP Exotica WG 2004-03, ALEPH 2004-007, DELPHI 2004-033 CONF 708, L3 Note 2798, OPAL Technical Note TN743. http://lepexotica.web.cern.ch/LEPEXOTICA/

18. G.F. Giudice, T. Plehn, A. Strumia, Nucl. Phys. B 706, 455 (2005)

19. L. Randall, R. Sundrum, Phys. Rev. Lett. 83, 3370 (1999)

20. P. Aarnio et al. (DELPHI Collaboration), Nucl. Instrum. Methods A 303, 233 (1991)

21. P. Abreu et al. (DELPHI Collaboration), Nucl. Instrum. Methods A 378, 57 (1996)

22. D. Karlen, Nucl. Phys. B 289, 23 (1987)

23. T. Sjöstrand et al., Comput. Phys. Commun. 135, 238 (2001)

24. F.A. Berends, P. Daverveldt, R. Kleiss, Comput. Phys. Commun. 40, 271 (1986), 285 and 309

25. T. Alderweireld et al., CERN Report 2000-009, ed. by G. Passarino, R. Pittau, S. Jadach (2000), p. 219

26. F.A. Berends, R. Gastmans, Nucl. Phys. B 61, 414 (1973)

27. F.A. Berends, R. Kleiss, Nucl. Phys. B 186, 22 (1981)

28. F.A. Berends et al., Nucl. Phys. B 239, 395 (1984)

29. S. Jadach, B.F.L. Ward, Z. Was, Comput. Phys. Commun. 66, 276 (1991)

30. S. Jadach, B.F.L. Ward, Z. Was, Comput. Phys. Commun. 79, 503 (1994)

31. F.A. Berends, R. Pittau, R. Kleiss, Comput. Phys. Commun. 85, 437 (1995)

32. J. Fujimoto et al., Comput. Phys. Commun. 100, 128 (1997)

33. G. Montagna et al., Nucl. Phys. B 452, 161 (1995)

34. G. Montagna et al., Nucl. Phys. B 541, 31 (1999)

35. S. Jadach, B.F.L. Ward, Z. Was, Comput. Phys. Commun. 130, $260(2000)$

36. T.W. Anderson, An Introduction to Multivariate Analysis (New York, Wiley, 1958)

37. V.F. Obraztsov, Nucl. Instrum. Methods A 316, 388 (1992) [Erratum: Nucl. Instrum. Methods A 399500 (1997)]

38. O. Nicrosini, L. Trentadue, Nucl. Phys. B 318, 1 (1989) 